\title{
Estudo comparativo de diferentes métodos de extração de compostos bioativos de plantas alimentícias não convencionais (PANC)
}

Comparative study of different methods of extracting bioactive compounds from wild edible plants (WEP)

\section{Estudio comparativo de diferentes métodos de extracción de compuestos bioactivos de plantas} alimentarias no convencionales (PANC)

\section{Resumo}

As Plantas Alimentícias Não Convencionais (PANC) são espécies rústicas, cosmopolitas e normalmente de fácil adaptação edafoclimáticas. Possuem uma ou mais partes comestíveis e não estão normalmente incluídas em nossa dieta cotidiana. Grande parte dessas espécies podem apresentar diferentes atividades biológicas, principalmente como antioxidantes. O objetivo deste trabalho foi avaliar a capacidade antioxidante e quantificar os principais compostos fenólicos de frutos da Leandra regnellii Cogn. (pixirica), Solanum americanum Mill (maria-pretinha) e Phytolaca thyrsiflora Hill. (fitolaca), em três diferentes métodos de extração, quais sejam: 1) aquosa por refluxo, 2) aquosa por maceração e 3) hidroetanólica. A maior atividade antioxidante obtida foi para a L. regnellii, sob extrato hidroetanólico e por refluxo. Quanto ao teor de compostos fenólicos, os melhores resultados obtidos foram para a espécie L. regnellii em extrato por maceração, seguida da $P$. thyrsiflora em extrato utilizando sistema de refluxo. Quanto a quantificação dos compostos majoritários por HPLC, a espécie L. regnellii também apresentou o maior teor de compostos fenólicos no extrato hidroetanólico, destacando-se o ácido ferúlico, ácido gálico, naringina, epicatequina e hesperidina. Na espécie $P$. thyrsiflora, foram identificados ácido gálico e quercetina em extrato hidroetanólico e apigenina em extrato por maceração. S. americanum no extrato hidroetanólico, apresentou rutina, catequina, epicatequina e vitexina como principais componentes. O presente estudo mostra atividade antioxidante expressiva assim como a presença de compostos fenólicos que apresentam atividade biológica ressaltando a importância da escolha do método de extração para futuros usos destas espécies pela indústria alimentícia e farmacêutica.

Palavras-chave: Antioxidantes; Compostos fenólicos; Fitoquímicos; PANC.

\section{Abstract}

Wild Edible Plants (WEP) are rustic species, cosmopolitan and normally easily adaptable to edaphoclimatic. They have one or more edible parts and are not normally included in our everyday diet. Most of these species can have different biological activities, mainly as antioxidants. The objective of this work was to evaluate the antioxidant capacity and 
quantify the main phenolic compounds of Leandra regnellii Cogn. (pixirica), Solanum americanum Mill (mariapretinha) and Phytolaca thyrsiflora Hill. (fitolaca), in three different extraction methods, namely: 1) aqueous by reflux, 2 ) aqueous by maceration and 3 ) hydroethanolic. The highest antioxidant activity obtained was for L. regnellii, under hydroethanolic extract and under reflux. As for the content of phenolic compounds, the best results were obtained for the species $L$. regnellii in maceration extract, followed by $P$. thyrsiflora in extract using a reflux system. As for the quantification of major compounds by HPLC, the species L. regnellii also presented the highest content of phenolic compounds in the hydroethanolic extract, especially ferulic acid, gallic acid, naringin, epicatechin and hesperidin. In the species $P$. thyrsiflora, gallic acid and quercetin were identified in hydroethanolic extract and apigenin in maceration extract. $S$. americanum in the hydroethanolic extract, presented rutin, catechin, epicatechin and vitexin as main components. The present study shows expressive antioxidant activity as well as the presence of phenolic compounds that have biological activity, highlighting the importance of choosing the extraction method for future uses of these species by the food and pharmaceutical industry.

Keywords: Antioxidants; Phenolic compounds; Phytochemicals; WEP.

\section{Resumen}

Las Plantas Alimentarias No Convencionales (PANC) son rústicas, cosmopolitas y normalmente fácilmente adaptables a especies edafoclimáticas. Tienen una o más partes comestibles y normalmente no se incluyen en nuestra dieta diaria. La mayoría de estas especies pueden tener diferentes actividades biológicas, principalmente como antioxidantes. El objetivo de este trabajo fue evaluar la capacidad antioxidante y cuantificar los principales compuestos fenólicos de Leandra regnellii Cogn. (pixirica), Solanum americanum Mill (frijol negro) y Phytolaca thyrsiflora Hill. (fitoloca), en tres métodos de extracción diferentes, a saber: 1) acuoso por reflujo, 2) acuoso por maceración y 3) hidroetanólico. La mayor actividad antioxidante obtenida fue para L. regnellii, bajo extracto hidroetanólico y bajo reflujo. En cuanto al contenido de compuestos fenólicos, los mejores resultados se obtuvieron para la especie L. regnellii en extracto de maceración, seguida de $P$. thyrsiflora en extracto mediante sistema de reflujo. En cuanto a la cuantificación de compuestos mayores por HPLC, la especie L. regnellii también presentó el mayor contenido de compuestos fenólicos en el extracto hidroetanólico, especialmente ácido ferúlico, ácido gálico, naringina, epicatequina y hesperidina. En la especie $P$. thyrsiflora se identificaron ácido gálico y quercetina en extracto hidroetanólico y apigenina en extracto de maceración. S. americanum en el extracto hidroetanólico, presentó rutina, catequina, epicatequina y vitexina como componentes principales. El presente estudio muestra una expresiva actividad antioxidante así como la presencia de compuestos fenólicos que tienen actividad biológica, destacando la importancia de elegir el método de extracción para usos futuros de estas especies por parte de la industria alimentaria y farmacéutica.

Palabras clave: Antioxidantes; Compuestos fenólicos; Fitoquímicos; PANC.

\section{Introdução}

Ao longo do processo evolutivo das civilizações, o ser humano têm apresentado papel importante quanto a seleção e domesticação de espécies vegetais. Porém, com a evolução da indústria química, farmacêutica e alimentícia, a população passou a preferir substâncias sintéticas para o tratamento de patologias e uso como suprimento nutricional humano. O aumento da expectativa de vida, o estresse diário e a falta de tempo, junto com a busca crescente de um modelo de vida mais saudável tem contribuído para pesquisas que visam desenvolver "alimentos funcionais", "nutracêuticos" ou "suplementos dietéticos" (SantosBuelga et al., 2019). Com isto, parte da cultura popular ficou depreciada, havendo descrédito da fitoterapia e muitas espécies caíram em desuso (Kinupp et al., 2007). Além disso, a preferência por alimentos industrializados e de rápido preparo, têm implicado em grandes impactos para o meio ambiente, constituindo um grave fator de ameaça à soberania e segurança alimentar populacional, uma vez que resulta em maior dependência da população de alimentos industrializados provenientes do mercado, implicando em maiores custos de vida e baixa diversidade alimentar (Cunha et al., 2021).

As plantas alimentícias não convencionais (PANC), normalmente são plantas espontâneas, conhecidas também como ervas daninhas ou inços. Várias espécies são utilizadas, principalmente, por comunidades rurais e tribais desde os tempos préhistóricos como fonte de compostos bioativos. Estas espécies, sejam nativas ou não, têm características que propiciam a sua adaptação à ambientes antropizados, como áreas agrícolas ou urbanas, gramados, calçadas pavimentas (Cheptou et al., 2008; Del Tredici, 2010). O grupo das PANC fazem parte da cultura de várias comunidades tradicionais no Brasil (Kinupp \& Barros 2007; Nascimento et al., 2013; Leal et al., 2018).

O consumo de frutos autóctones, coloridos e ruderais, desempenham um papel importante na dieta humana em 
diferentes partes do planeta. Avaliações destas espécies podem contribuir para a descoberta de novos nutracêuticos oriundos dos compostos fenólicos, importantes na prevenção de doenças relacionadas ao metabolismo redox e inflamatório das células (Fillipin et al., 2008). Neste sentido, várias investigações científicas recentes têm reportado diversos compostos bioativos com propriedades antioxidantes presentes nas PANC, que desempenham grande importância na prevenção de doenças como o câncer, doenças cardíacas, osteoporose e doenças degenerativas (Maphosa \& Jideani, 2017; Prakash \& Sharma, 2014). Muitas destas espécies PANC, atribuem diversos benefícios para a saúde, podendo ser novas fontes de alimentos funcionais e propriedades nutracêuticas na dieta humana. (Boari, et al., 2013; Baldermann, et al., 2016; Petropoulos, et al., 2018).

O conhecimento do conteúdo de compostos bioativos e da capacidade antioxidante de frutas nativas negligenciadas, visa agregar valor comercial e industrial a estas espécies, contribuir com a conservação dos biomas e propor alternativas para o desenvolvimento de novos fármacos. A família Melastomataceae possui cerca de 4.500 espécies, distribuídas em 150 gêneros em todo o mundo, sendo Miconia e Leandra, os mais representativos no Brasil, principalmente pelo alto grau de endemismo (Renner et al., 2010; Goldenberg et al., 2009). Os frutos da L. regnelli são consumidos pela população rural, podendo ser considerada como "mirtilo brasileiro", devido a sua coloração e morfologia muito semelhante. Possui também grande importância ecológica devido a sua apreciação por diversas espécies da fauna silvestre, sendo amplamente utilizada em programas de recuperação de áreas degradadas (Primack \& Rodrigues, 2001).

A espécie S. americanum, pertencente à família Solanaceae, é uma PANC geralmente relacionada às áreas antropizadas, ricas em nitrogênio e úmidas, como solos agrícolas, pomares, hortas, jardins e terrenos baldios. Esta espécie ruderal é muito utilizada no Brasil em pratos diversos, seus frutos são considerados como iguaria, rico em antocianinas e antioxidantes (Kinupp et al., 2007). Há estudos relacionados à composição centesimal das suas folhas cozidas, pois são muito consumidas na Guatemala, El Salvador, México e China, pelo seu teor de lipídeos, proteínas e minerais como o cálcio, ferro, magnésio e potássio (Kinupp et al., 2014). Pesquisas científicas relacionadas aos seus frutos não foram encontradas até o presente momento. A espécie P. thyrsiflora, família Phytolaccaceae, é uma espécie utilizada, principalmente, pela população do norte do Brasil. Suas raízes e frutos são considerados purgativos, porém suas folhas e brotos são utilizados na culinária regional, após cozimento adequado, escaldando pelos menos três vezes (Kinupp et al., 2014). Os frutos já foram amplamente utilizados popularmente como corante, por isso também é conhecida como tinge-ovos (FRSB, 2001; Treyvaud et al., 2000).

Muitos fatores podem afetar a eficiência da solubilidade e extração dos metabólitos secundários das plantas, por exemplo, tempo de extração, solvente, proporção de amostra frente ao soluto e temperatura (Peng et al., 2019). A otimização dos métodos de extração podem elucidar perfis metabólicos diversificados e com maior eficiência para uma mesma espécie. Neste sentido, considerando o potencial farmacológico e alimentício das PANC, propomos investigar três espécies ruderais, quais sejam, Leandra regnellii Cogn. (pixirica), Solanum americanum Mill (maria-pretinha) e Phytolaca thyrsiflora Hill. (fitolaca), todas submetidas a diferentes métodos extrativos com o objetivo de contribuir para a descoberta de novas identidades químicas com potencial farmacológico e apoiar pesquisas futuras que enfatizem o potencial da biodiversidade brasileira. Para a realização deste estudo, foram analisados o teor de compostos fenólicos totais, a capacidade de sequestro do radical DPPH· e a quantificação de compostos por HPLC-UV, determinados por técnicas espectrofotométricas.

\section{Metodologia}

\section{Material vegetal}

Os frutos das três espécies avaliadas L. regnellii (pixirica), S. americanum (maria-pretinha) e P. thyrsiflora (fitolaca) (Figura 1) foram coletados nos meses de setembro e outubro do ano de 2016, no município de Caxias do Sul, sendo identificadas, catalogadas e registradas no Herbário da Universidade de Caxias do Sul (HUCS), conforme descritos na Tabela 1. Os frutos foram separados, pesados e imediatamente congelados para posteriores análises. Este estudo trata-se de uma pesquisa 
experimental explicativa, realizado junto ao laboratório de Estresse Oxidativo e Antioxidantes do Instituto de Biotecnologia da Universidade de Caxias do Sul. A natureza da investigação é básica e a sua abordagem é quali-quantitativa, cujo método de procedimento é experimental (Pereira et al., 2018).

Tabela 1 - Descrição botânica e registro adequado das espécies amostradas.

\begin{tabular}{|c|c|c|c|c|}
\hline Nome científico & Nome comum & Família & Local de coleta & HUCS \\
\hline Solanum americanum Mill. & maria-pretinha & Solanaceae & $\mathrm{S}-29^{\circ} 00^{\prime} 11.0^{\prime \prime}, \mathrm{W}-050^{\circ} 54^{\prime} 06.8^{\prime \prime} \mathrm{N}$ & 45802 \\
\hline $\begin{array}{c}\text { Phytolacca thyrsiflora Fenzl. ex } \\
\text { J. A. Schmidt }\end{array}$ & fitolaca & Phytolaccaceae & S- $29^{\circ} 09^{\prime} 40.7^{\prime \prime}, \mathrm{W}-051^{\circ} 08^{\prime} 45.0^{\prime \prime} \mathrm{N}$ & 45804 \\
\hline Leandra regnellii (Triana) Cogn. & pixirica & Melastomataceae & S- $29^{\circ} 09^{\prime} 47.7^{\prime \prime}, \mathrm{W}-051^{\circ} 08^{\prime} 43.6^{\prime \prime} \mathrm{N}$ & 45803 \\
\hline
\end{tabular}

Fonte: Autores.

Figura 1 - Frutos das três espécies amostradas. A) Phytolacca thyrsiflora (fitolaca); B) Solanum americanum (maria-pretinha); C) Leandra regnellii (pixirica).

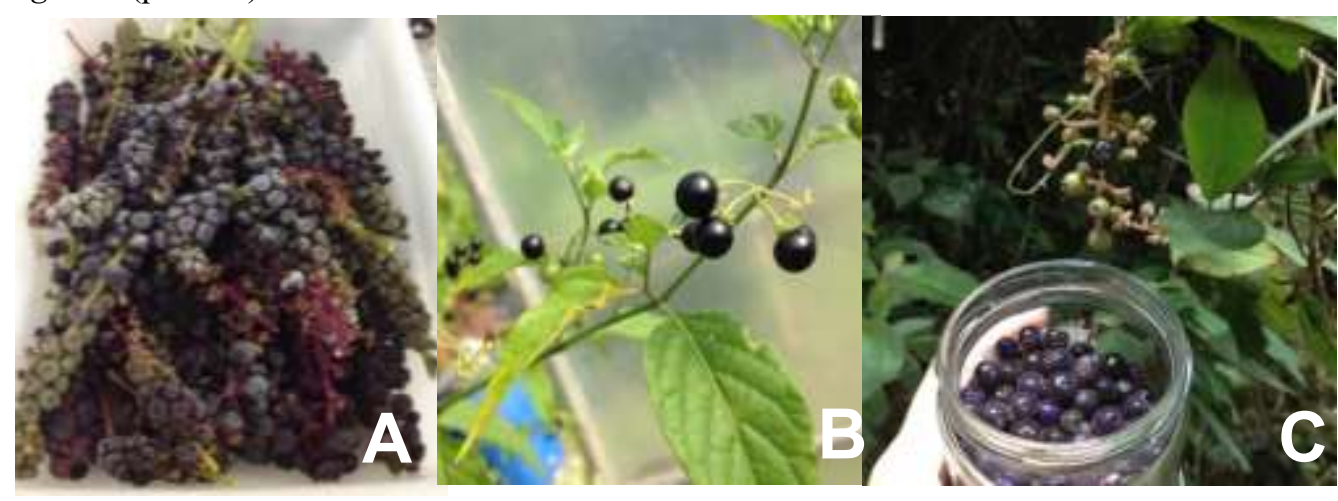

Fonte: Autores.

\section{Processamento dos frutos}

Foram utilizados $100 \mathrm{~g}$ dos frutos da pixirica (L. regnellii), maria-pretinha (S. americanum) e fitolaca (P. thyrsiflora). Os mesmos foram macerados e triturados utilizando um mixer, obtendo uma mistura homogênea. Dos macerados foram preparados três extratos diferentes à $5 \%(\mathrm{p} / \mathrm{v})$ de frutos, sendo eles: 1) Extrato aquoso por maceração; 2) Extrato aquoso utilizando sistema de refluxo; 3) Extrato Hidroetanólico à 80\%. Os extratos hidroetanólico e maceração permaneceram por 15 minutos no escuro, sendo agitados de 5 em 5 minutos, à temperatura ambiente, posteriormente acondicionados em local ao abrigo da luz. O extrato sob sistema de refluxo, foi fervido em condensador a $100^{\circ} \mathrm{C}$, sendo mantido a fervura por 15 minutos, posteriormente resfriado até $20^{\circ} \mathrm{C}$. Após esse período os extratos foram filtrados a vácuo sob temperatura ambiente para a realização dos ensaios.

\section{Avaliação da atividade antioxidante pelo método do sequestro do radical 2,2-difenil-1-picril-hidrazila (DPPH)}

A atividade antioxidante in vitro dos extratos foi avaliada através dos métodos DPPH; descrito por Yamaguchi et al., (1998). No método DPPH· foi determinada a capacidade do extrato em doar hidrogênio ao radical livre DPPH·, modificando, assim, a coloração da solução de roxo para amarelo. Para a realização deste ensaio, $100 \mu \mathrm{L}$ dos diferentes extratos hidroetanólico (etOH) à $80 \%$, maceração e refluxo, foram adicionados a $400 \mu \mathrm{L}$ de uma solução tampão Tris- $\mathrm{HCl}(100 \mathrm{mM}, \mathrm{pH} 7,0)$. A esta mistura foi adicionada $500 \mu \mathrm{L}$ de uma solução etanólica de DPPH· $(500 \mu \mathrm{M})$. Após, as amostras foram agitadas e os tubos permaneceram em repouso por 20 minutos ao abrigo da luz. Para o padrão, o extrato foi substituído por água destilada. Os valores de absorbância foram mensurados espectrofotometricamente a $517 \mathrm{~nm}$ e os dados expressos em \% do sequestro do radical. 


\section{Determinação do teor de compostos fenólicos totais}

A determinação do teor de Compostos Fenólicos Totais foi realizada através da metodologia de Folin-Ciocalteau, com modificações, descrita por Singleton et al., (1965), onde foi utilizado $100 \mu \mathrm{L}$ dos extratos hidroetanólico (etOH) à $80 \%$, maceração e de refluxo, diluídos em $500 \mu \mathrm{L}$ do reagente Folin $1 \mathrm{~N}$ e $400 \mu \mathrm{L}$ de carbonato de sódio $\left(\mathrm{Na}_{2} \mathrm{CO}_{3}\right)(7,5 \%$ p/v). Após, as amostras foram agitadas e permaneceram em repouso por 30 minutos ao abrigo da luz. Para a curva padrão, utilizou-se ácido gálico em concentrações que variaram de 0,025 a $0,75 \mathrm{mg} / \mathrm{mL}$. As absorbâncias foram determinadas a $765 \mathrm{~nm}$, em espectrofotômetro (UV-1700, Shimadzu Pharmaspec). Os resultados foram expressos em mg\% de equivalentes de ácido gálico $(\mathrm{mg} \%$ de $\mathrm{EAG})$.

\section{Validação do método analítico}

Para validação do método analítico empregado, foram avaliados a seletividade, linearidade e precisão. Os limites de detecção e de quantificação também foram calculados de acordo com Ribani et al., (2004). A seletividade foi verificada através de comparação dos tempos de retenção dos picos dos compostos padrões injetados individualmente. Para avaliar a linearidade do método, foram construídas curvas analíticas de cada padrão em triplicata, sob seis concentrações diferentes. A precisão do método foi verificada pela repetibilidade da mesma amostra analisada em nove replicatas, com valores expressos através da média e desvio padrão relativo. Para o cálculo dos limites de detecção (LD) e de quantificação (LQ) dividiu-se o coeficiente linear pelo coeficiente angular da curva analítica, para cada um dos padrões. Estes resultados foram multiplicados por 3 para LD e por 10 para LQ.

\section{Análise de compostos fenólicos por cromatografia líquida de alta eficiência - HPLC-UV}

Os extratos vegetais foram analisados em equipamento HPLC marca HP modelo 1100, coluna Lichrospher RP18 (5 $\mu \mathrm{m})$ acoplada a detector UV a 210nm (HPLC-UV) e sistema quaternário de bombas. A análise em fase reversa foi constituída de: solvente A - água Milli-Q com 1\% de ácido fosfórico e solvente B - Acetonitrila. O sistema de bombeamento da fase móvel foi gradiente, com $90 \%$ do solvente A de 0 a $5 \mathrm{~min}, 60 \%$ de A de 5 a 40min e 90\% de A de 45 a 50 min. O fluxo padrão foi mantido a $0,5 \mathrm{~mL} / \mathrm{min}$ de acordo com Morelli (2010). O volume de injeção foi de 10 $\mu \mathrm{L}$. As amostras foram filtradas em membranas de Nylon de $0,45 \mu \mathrm{m}$ de diâmetro de poro. Os compostos fenólicos foram identificados de acordo com sua ordem de eluição e por comparação de seu tempo de retenção com aqueles de seus padrões puros. A quantificação foi realizada pelo método de padronização externa, através da correlação da área $\left(m A U^{*} s\right)$ do pico do composto à curva padrão realizada com cada padrão avaliado (ácido gálico, epigalocatequina, catequina, epicatequina, epigalocatequina galato, rutina, vitexina, ácido ferulico, naringina, hesperidina, miricetina, resveratrol, quercetina e apigenina).

\section{Resultados e Discussão}

\section{L. regenelllii apresenta maior capacidade antioxidante}

De acordo com Wu et al., (2013), algumas frutas, especialmente aquelas de cor escura (uva, mirtilo, ameixa, cereja, amora, jabuticaba), têm sido reportadas como "superfrutas" devido à sua alta capacidade antioxidante. Este fato é extremamente relevante para a saúde humana, pois a capacidade de sequestrar radicais instáveis é considerada como atividade importante para a promoção da saúde humana através da adição de espécies ricas em compostos fenólicos à dieta, especialmente frutos ricos em flavonóides. Atualmente se faz necessário uma mudança radical no cardápio cotidiano da população, sendo assim, o consumo de espécies PANC, além de contribuir para o enriquecimento nutricional da nossa dieta usual, também apresenta seguridade no seu consumo, pois não demandam do uso de agroquímicos para a sua produção, melhorando a qualidade da alimentação de famílias inseridas tanto no meio rural, quanto urbano (Barbosa et al., 2021). 
Entre os frutos amostrados (Figura 2), nas concentrações propostas para este estudo, foi analisado uma porcentagem de varredura do radical $\mathrm{DPPH}^{*}$ que variou de $37 \%$ a $88 \%$. Foi verificada diferença estatística tanto entre as espécies como também quanto ao método de extração, destacando-se a pixirica (L. regnellii) com maior atividade antioxidante quando extraída por refluxo e etanol $80 \%$. As frutas e vegetais de coloração arroxeada têm se apresentado comercialmente atrativo devido ao seu teor de compostos, principalmente pela presença das antocianinas. As "super-frutas" e os vegetais como a cebola-roxa e o repolhoroxo possuem flavonoides de interesse para a indústria farmacêutica, os quais podem viabilizar o desenvolvimento de produtos naturais anticancerígenos promissores (Middleton, et al., 2000; Sandhar, et al., 2011). Nesse sentido, é imprescindível a descoberta de novas fontes que sejam viáveis economicamente e principalmente, nativas da região, facilitando o acesso à população e pesquisadores.

Figura 2 - Atividade antioxidante de L. regnelli, S. americanum e P. thyrsiflora em três diferentes tipos de extração. Os valores são expressos em média \pm desvio padrão. A análise estatística foi realizada entre todas as plantas, onde letras diferentes indicam valores significativamente diferentes de acordo com a análise de variância ANOVA e pós teste de Tukey. Significância estatística é de $\mathrm{p}<0,05$.

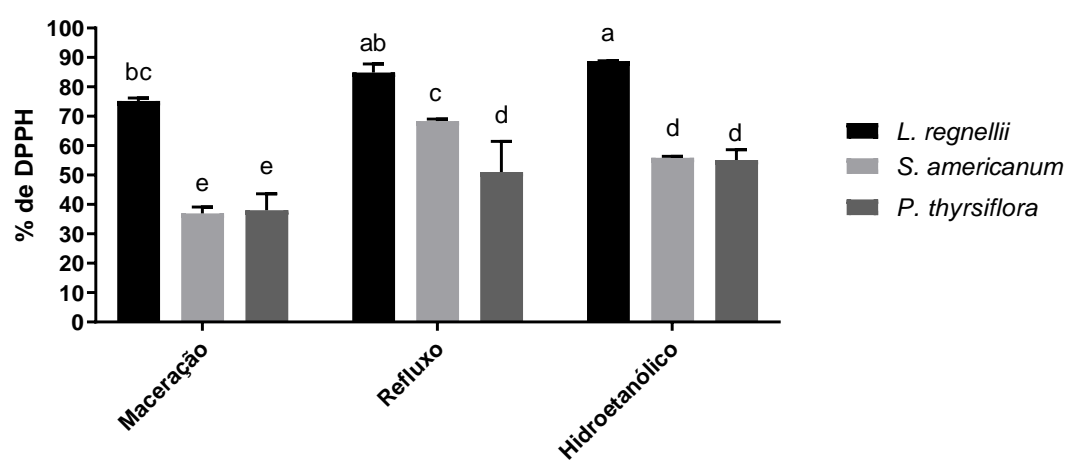

Fonte: Autores.

A atividade antioxidante já tem sido reportada pela literatura para a família Melastomataceae. Estudos com extratos do pólen coletado de colmeias de Apis melifera demonstraram 97\% a presença de esporos de espécies do gênero Miconia, os quais apresentaram alto teor de compostos fenólicos, boa capacidade antioxidante e atividade anti-hemolítica (Araújo et al., 2017). O uso conservacionista de espécies ruderais contribui também para a conservação da fauna local. Aves e pequenos insetos frequentemente buscam estas espécies como base alimentar, favorecendo a cadeia trófica. Outros estudos demonstram atividade anti-inflamatória, antioxidante, anti-mutagênicas, anti-nociceptivas, digestivas e hepatoprotetora para o extrato metanólico de frutos de Miconia albicans, atividade atribuída pela presença de alto teor de compostos fenólicos (Corrêa et al., 2021). O extrato etanólico utilizando folhas de M. albicans também apresentou capacidade em reduzir os sintomas de artrite reumatoide em ratos (Quintans-Júnior et al., 2020). Ferreira-Gomes e colaboradores (2021), avaliaram atividade biológica, antioxidante e compostos fitoquímicos de extrato aquoso das folhas de Miconia chamissois resultados indicaram a presença de flavonóides e polifenóis incluindo rutina, isoquercitrina e derivados de vitexina.

Extratos metanólicos dos frutos de Solanum nigrum têm sido reportados pela sua alta capacidade antioxidante, principalmente devido à presença de antocianinas cis-trans, que apresentam capacidade de sequestro de radicais dezenas de vezes maior que a vitamina C (Meng et al., 2020). A ação biológica da fração clorofórmica de extratos dos frutos de S. nigrum, também apresentaram boa capacidade antioxidante e ação antiproliferativa significativa contra linhagens de células tumorais, devido à indução à apoptose (Khan et al., 2016). Hameed e Akhtar (2018) também mostram em seu estudos que os extratos de S. nigrum 
apresentam atividade marcante de inibição da enzima tirosinase e atividade antioxidante, atribuídas a presença dos polifenóis e flavonóides. A espécie $S$. nigrum é muito próxima filogeneticamente da $S$. americanum, avaliada neste estudo logo, podemos inferir que estas atividades também podem ser atribuídas para esta espécie em questão, bem como a M. albicans e a L. regenellii.

Há somente três estudos quanto às espécies próximas filogeneticamente da $P$. thyrsiflora. Nabavi et al., (2009) avaliaram a capacidade antioxidante do extrato metanólico de frutos de Phytolacca americana, demonstrando capacidade de sequestro do radical DPPH $\left(\mathrm{IC}_{50}=62 \mu \mathrm{g} / \mathrm{mL}\right)$, Óxido nítrico $(3,2 \mathrm{mg} / \mathrm{ml})$ e $\mathrm{H}_{2} \mathrm{O}_{2}\left(\mathrm{IC}_{50}=245,3 \mu \mathrm{g} / \mathrm{mL}\right)$. Esta atividade foi significativamente maior do que a da vitamina $\mathrm{C}(\mathrm{p}<0,01)$. O extrato apresentou alto teor de fenólicos totais. Ullah et al., (2015) avaliou o extrato metanólico das partes aéreas da Phytolacca latbenia. Neste estudo também foi confirmado o potencial antioxidante da espécie através do aumento da atividade das enzimas antioxidantes endógenas em sementes (in vitro). E por fim, Zheleva-Dimitrova (2013) avaliou o extrato metanólico de folhas e frutos de Phytolacca americana, demonstrando capacidade antioxidante por $\mathrm{DPPH}^{\cdot}\left(\mathrm{IC}_{50}=88,79 \mu \mathrm{g} / \mathrm{mL}\right)$ e ABTS ${ }^{+}(18,43 \mu \mathrm{g} / \mathrm{mL})$ para folhas e DPPH${ }^{*}\left(\mathrm{IC}_{50}=412,06 \mu \mathrm{g} / \mathrm{mL}\right)$ e $\mathrm{ABTS}^{+}(112,49 \mu \mathrm{g} / \mathrm{mL})$, para frutos. Quanto ao teor de compostos fenólicos totais as folhas apresentaram 215,79mg de eq. pirogalol/g e os frutos 174,76mg de eq. pirogalol/g de extrato seco, demonstrando boa capacidade antioxidante e teor de compostos fenólicos totais.

Os compostos fenólicos são metabólitos secundários que estão envolvidos na defesa das plantas contra a radiação ultravioleta ou agressão por patógenos, parasitas e predadores, além de contribuir para a coloração e propriedades organolépticas das espécies (Tufarelli et al, 2017). Estes compostos possuem propriedades antioxidantes e efeitos biológicos marcantes na prevenção de várias doenças associadas ao estresse oxidativo, como o câncer, ateroesclerose, diabetes Mellitus e doenças neurodegenerativas (Dai \& Mumper, 2010). Os compostos fenólicos são um grupo de fitoquímicos classificados como flavonóides (flavonóis, flavonóis, flavonas, flavanonas, isoflavonas e antocianinas) e moléculas não-flavonóides (ácidos fenólicos, ácidos hidroxicinâmicos, lignanos, estilbenos e taninos), que podem atribuir diversos benefícios à saúde pública (Di Lorenzo, et al., 2021).

Para a análise de compostos fenólicos totais das três PANC amostradas (Figura 3), observamos diferenças significativas quanto ao método de extração e espécie analisada, onde a planta que destacou-se novamente foi a L. regenellii, em extrato por maceração e por refluxo, sendo considerada, neste estudo, a espécie mais promissora, que servirá de base para futuras pesquisas mais detalhadas. Apesar dos compostos fenólicos apresentarem inúmeros benefícios à população, os estudos mediante aos mecanismos de ação ainda são limitados, pois poucas pesquisas demonstram a real interação entre os compostos consumidos na dieta e a sua absorção efetiva pela microbiota intestinal. Estudos recentes têm reportado a estimativa do consumo diário destes compostos pela população em cerca de $0,9 \mathrm{~g}$, através da ingestão de chás, café, sucos, vinho tinto, frutas e vegetais. Porém a correlação com a biodisponibilidade desses compostos no organismo não foi avaliada (Di Lorenzo et al., 2021). 
Figura 3 - Quantificação de compostos fenólicos totais de L. regnelli, S. americanum e P. thyrsiflora, em três diferentes tipos de extração. Os valores são expressos em Média \pm DP. A análise estatística foi realizada entre todas as plantas, onde letras diferentes indicam valores significativamente diferentes de acordo com a análise de variância ANOVA e pós teste de Tukey. Significância estatística é de $\mathrm{p}<0,05$.

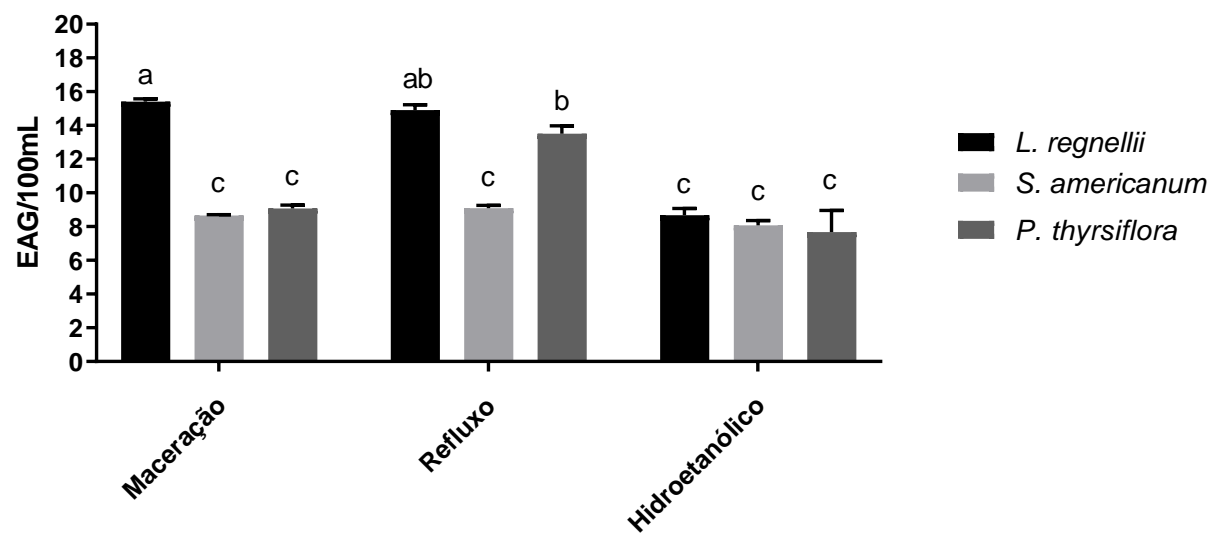

Fonte: Autores.

Investigações fitoquímicas para o gênero Solanum demonstram a presença de compostos como saponinas esteróides, alcalóides esteróides, terpenos, flavonóides, lignanas, esteróis, compostos fenólicos, cumarinas, entre outros (Kaunda \& Zhang, 2019). Diversas espécies pertencentes à família Solanaceae apresentam atividades farmacológicas de interesse, como citotoxicidade para diferentes tipos de tumores, como câncer de mama, câncer colorretal e câncer de próstata, um bom exemplo é atribuído ao consumo de tomate (Kaunda \& Zhang, 2019). Além de muitas outras atividades atribuídas às Solanaceaes como, ação analgésica, anti-helmíntica, antialérgica, antianêmica, antiasmática, antibacteriana, anticâncer, anticonvulsivante, antidepressiva, antidiabética, antifúngica, anti-histamínica, anti-hipertensiva, antiinflamatória, antileishmania, antimelanogenético, antimoluscicida, antinociceptivo, antipsoriático, antiplasmódico, antiprotozoário, antitripanossomal, antiurolitiatico, antiviral, cardio-vascular, diurético, hepatoprotetivo, hipolipidêmico, mosquito-larvicida, esquito-larvicida, nepolipidêmico atividades vasorrelaxantes (Kaunda \& Zhang, 2019).

Estudos fitoquímicos de Morais et al., (2020) utilizando extrato de frutos maduros de Solanum lycocarpum demonstraram capacidade antioxidante, anti-inflamatória e antinociceptiva, atribuídas principalmente à presença de alcalóides, como solasodina e peiminina, além de ácidos cafeoilquínicos, indicado pelos autores no tratamento de processos inflamatórios e no combate de dores. Extratos dos frutos verdes de Solanum nigrum apresentaram ações anti-inflamatória e anti-proliferativa em modelo celular, impactando também na supressão da produção de óxido nítrico induzido por lipopolissacarídeo em macrófagos (Xiang et al., 2019). Yang et al., (2021) realizaram análises fitoquímicas aliadas a ferramentas da bioinformática para identificar os principais compostos presentes nos extratos de frutos de S. nigrum, e também, identificaram os principais alvos terapêuticos para o tratamento de tumores através de molecular networking. Foram identificados neste estudo 170 compostos, entre eles 114 alcalóides esteroidais, dentre estes 14 foram analisados por molecular docking, demonstrando provável ação antitumoral, agindo através de 99 alvos biológicos distintos. Vale ressaltar que a atividade antioxidante e funções biológicas para as três espécies PANC analisadas neste estudo não foram relatadas até o presente momento.

\section{Extrações utilizando solvente aquoso combinado com etanol promove maior solubilidade de compostos fenólicos}

Para validação do método empregado, avaliou-se a seletividade dos padrões utilizados. Após a separação das amostras dos padrões (Figura 4), foram realizadas curvas de calibração (Tabela 2), para estabelecer a análise em faixa linear. Observamos 
através do $\mathrm{R}^{2}$ de cada curva e de seus limites de detecção e quantificação, que os resultados foram adequados e satisfatórios para a metodologia empregada.

Tabela 2 - Parâmetros das curvas de calibração dos compostos avaliados ( $\mu \mathrm{g} \mathrm{mL-1})$.

\begin{tabular}{|c|c|c|c|c|c|}
\hline $\begin{array}{c}\text { Compostos } \\
\text { fenólicos }\end{array}$ & Coeficiente $\left(\mathbf{R}^{2}\right)$ & $\begin{array}{c}\text { Níveis de } \\
\text { concentração }\end{array}$ & $\begin{array}{c}\text { Curva de } \\
\text { calibração }\end{array}$ & $\begin{array}{c}\text { Limite de } \\
\text { detecção (LD) }\end{array}$ & $\begin{array}{c}\text { Limite de } \\
\text { quantificação (LQ) }\end{array}$ \\
\hline Ácido gálico & 0,9999 & $0,1-30$ & $\mathrm{Y}=69,95 \mathrm{x}+0,66$ & 0,03 & 0,10 \\
\hline Catequina & 0,9982 & $0,06-16$ & $\mathrm{Y}=179,43 \mathrm{x}-30,56$ & 0,02 & 0,06 \\
\hline Epicatequina & 0,9995 & $0,1-50$ & $\mathrm{Y}=123,22 \mathrm{x}-54,57$ & 00,04 & 0,09 \\
\hline Rutina & 0,9996 & $0,1-25$ & $\mathrm{Y}=29,71 \mathrm{x}+1,19$ & 0,04 & 0,19 \\
\hline Vitexina & 0,9972 & $0,2-60$ & $\mathrm{Y}=38,89 \mathrm{x}+1,62$ & 0,06 & 0,11 \\
\hline Ácido ferúlico & 0,9990 & $0,1-50$ & $\mathrm{Y}=113,61 \mathrm{x}-11,98$ & 0,03 & 0,09 \\
\hline Naringina & 0,9926 & $0,1-16$ & $\mathrm{Y}=55,59 \mathrm{x}+45,30$ & 0,03 & 0,08 \\
\hline Hesperedina & 0,9993 & $0,1-12,5$ & $\mathrm{Y}=40,76 \mathrm{x}+4,50$ & 0,02 & 0,18 \\
\hline Miricetina & 0,9972 & $0,2-50$ & $\mathrm{Y}=105,82 \mathrm{x}-64,59$ & 0,05 & 0,10 \\
\hline Resveratrol & 0,9999 & $0,1-12,5$ & $\mathrm{Y}=63,76 \mathrm{x}+0,27$ & 0,03 & 0,12 \\
\hline Quercetina & 0,9999 & $0,1-25$ & $\mathrm{Y}=64,11 \mathrm{x}-0,68$ & 0,04 & 0,08 \\
\hline Apigenina & 0,9999 & $0,1-50$ & $\mathrm{Y}=85,62 \mathrm{x}+16,16$ & 0,02 & \\
\hline
\end{tabular}

Fonte: Autores.

No que tange a elucidação e quantificação dos compostos fenólicos, foram avaliados 14 padrões por HPLC-UV, quais sejam, ácido gálico, epigalocatequina, catequina, epicatequina, epigalocatequina galato, rutina, vitexina, ácido ferulico, naringina, hesperidina, miricetina, resveratrol, quercetina e Apigenina (Figura 4). A metodologia analítica desenvolvida permitiu a análise quantitativa de 10 compostos antioxidantes majoritários entre as três espécies nas diferentes extrações. A extração hidroetanólica foi a mais rica em compostos fenólicos para as três espécies analisadas (Tabela 3). Os compostos encontrados nas três espécies variaram conforme o método de extração e planta, como esperado. 
Research, Society and Development, v. 10, n. 17, e190101724210, 2021

(CC BY 4.0) | ISSN 2525-3409 | DOI: http://dx.doi.org/10.33448/rsd-v10i17.24210

Figura 4: Perfil cromatográfico dos padrões (ácido gálico (1), epigalocatequina (2), catequina (3), epicatequina (4), epigalocatequina galato (5), rutina (6), vitexina (7), ácido ferulico (8), naringina (9), hesperidina (10), miricetina (11), resveratrol (12), quercetina (13) e Apigenina (14)).

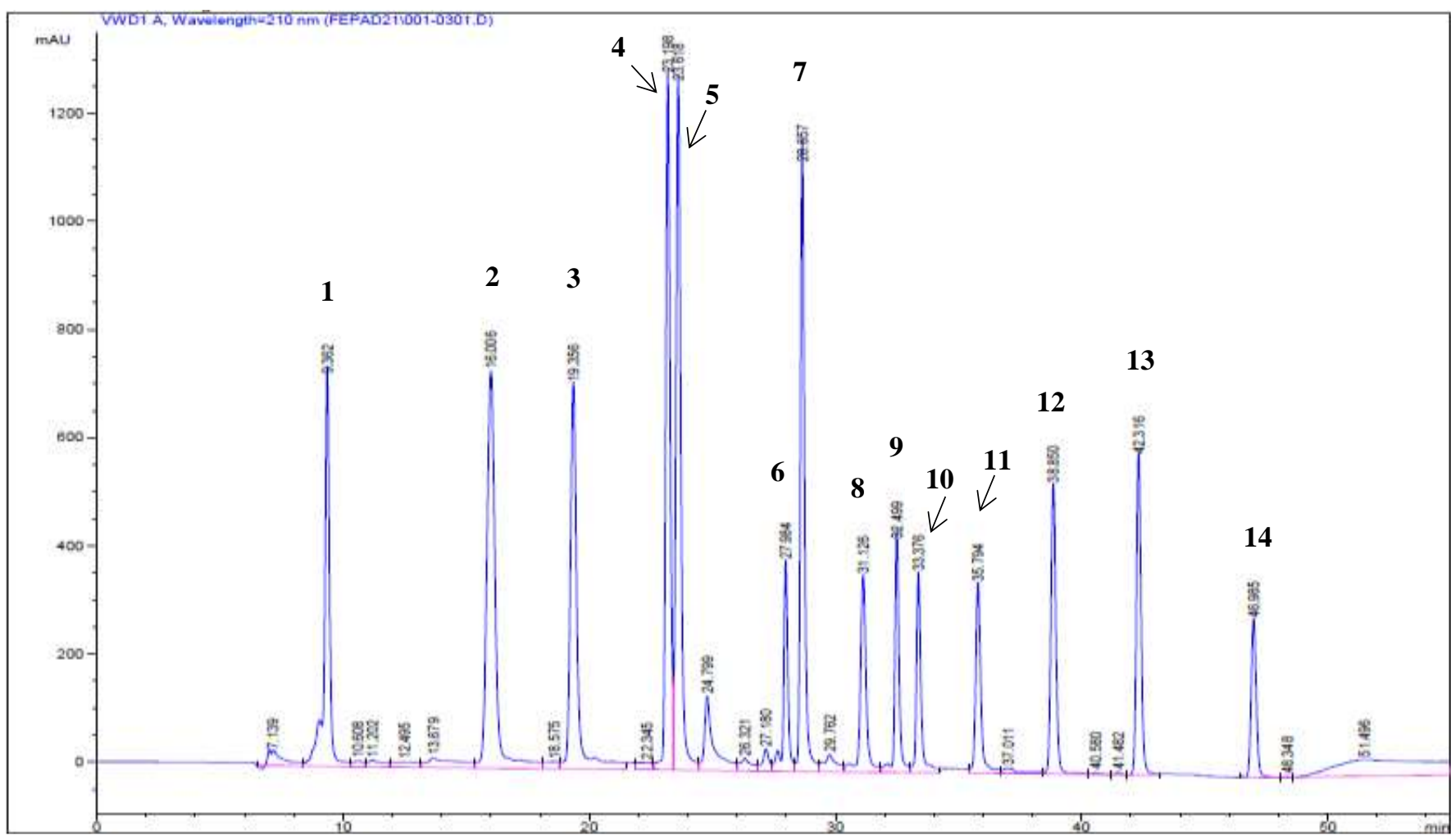

Fonte: Autores 
Research, Society and Development, v. 10, n. 17, e190101724210, 2021

(CC BY 4.0) | ISSN 2525-3409 | DOI: http://dx.doi.org/10.33448/rsd-v10i17.24210

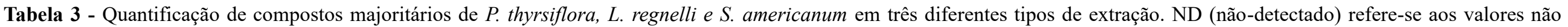
amostrados acerca dos métodos utilizados. Os valores são expressos em média e $\mu \mathrm{g} / \mathrm{ml}$.

\begin{tabular}{|c|c|c|c|c|c|c|c|c|c|c|c|}
\hline Planta & Extratos & Rutina & Catequina & $\begin{array}{c}\text { Ác. } \\
\text { Gálico }\end{array}$ & Epicatequina & Vitexina & Hesperidina & Quercetina & Naringina & $\begin{array}{c}\text { Ác. } \\
\text { Ferúlico }\end{array}$ & Apigenina \\
\hline \multirow{3}{*}{ P. thyrsiflora } & Maceração & 1,37 & 2,037 & 7,572 & ND & 1,052 & ND & ND & ND & ND & 3,301 \\
\hline & HidroetOH & 2,29 & 2,015 & 12,342 & ND & 3,443 & ND & 8,052 & ND & ND & 1,623 \\
\hline & Refluxo & 1,359 & 1,938 & 7,290 & ND & 0,954 & ND & ND & ND & ND & 2,991 \\
\hline \multirow{3}{*}{ S. americanum } & Maceração & 0,810 & 1,401 & ND & 1,170 & 0,743 & ND & ND & ND & ND & ND \\
\hline & HidroetOH & 17,939 & 12,766 & 17,94 & 10,403 & 7,420 & ND & ND & ND & ND & ND \\
\hline & Refluxo & 0,734 & 2,423 & ND & 1,807 & 1,003 & ND & ND & ND & ND & ND \\
\hline \multirow{3}{*}{ L. regnellii } & Maceração & ND & 1,045 & 1,447 & 1,309 & ND & ND & ND & ND & ND & ND \\
\hline & HidroetOH & ND & 1,875 & 11,461 & 2,584 & 1,556 & 2,033 & ND & 9,91 & 26,078 & ND \\
\hline & Refluxo & ND & 1,166 & 1,544 & 0,661 & ND & ND & ND & ND & ND & ND \\
\hline
\end{tabular}

Fonte: Autores. 
Para a espécie $P$. thyrsiflora, 6 compostos foram identificados, sendo os que apresentaram maior concentração foram ácido gálico $(12,3 \mu \mathrm{g} / \mathrm{ml})$ e quercitina $(8 \mu \mathrm{g} / \mathrm{ml})$ em extrato hidroetanólico e apigenina $(3,3 \mu \mathrm{g} / \mathrm{ml})$ em extrato aquoso. Para a $L$.

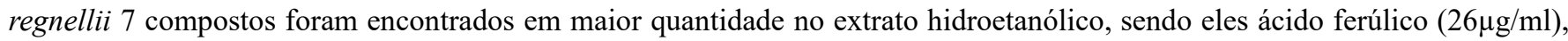
ácido gálico $(11,46 \mu \mathrm{g} / \mathrm{ml})$, naringina $(9,91 \mu \mathrm{g} / \mathrm{ml})$, epicatequina $(2,58 \mu \mathrm{g} / \mathrm{ml})$ e hesperidina $(2 \mu \mathrm{g} / \mathrm{ml})$. Para $S$. americanum a extração que melhor apresentou análise e identificação de compostos foi também a hidroetanólica, onde quantificamos rutina $(17,93 \mu \mathrm{g} / \mathrm{ml})$, catequina $(12,76 \mu \mathrm{g} / \mathrm{ml})$, epicatequina $(10,40 \mu \mathrm{g} / \mathrm{ml})$ e vitexina $(7,42 \mu \mathrm{g} / \mathrm{ml})$.

Métodos de extração e solventes eficientes, de polaridade específica, para a caracterização dos compostos fenólicos implicam diretamente aos baixos custos para a indústria farmacêutica e alimentícia. Por se tratarem de moléculas extremamente complexas, ainda não há um método específico para a extração destes compostos bioativos, no que tange tempo, solvente e equipamentos (Dai \& Mumper, 2010). Solventes, como metanol, etanol, acetona, acetato de etila e suas combinações têm sido usados para a extração de fenólicos de materiais vegetais, muitas vezes com diferentes proporções de água (Xu \& Chang, 2006). Porém, a maioria destes solventes podem apresentar toxicidade aos organismos e ambiente, o qual não observa-se ao utilizarmos a água. Neste estudo, observamos que as extrações com solvente aquoso puro, tanto a quente - utilizando sistema de refluxo, quanto a frio - utilizando maceração foram os melhores métodos para a extração das moléculas bioativas com atividade antioxidante. Demonstrando que o uso de solventes aquosos atribuem uma boa extração para os compostos fenólicos presentes nas plantas. Sendo atribuído também a técnica milenar do consumo de chás para tratamento de patologias diversas.

Um dos benefícios do consumo de frutas e outros vegetais é geralmente atribuído aos flavonóides, que podem ser encontradas em vinho tinto, suco de uva, maçã, cebola, alface, cereja, chá-preto, chá-verde, soja (Rissi et al., 2012). São compostos antioxidantes capazes de modular as funções mitocondriais, promovendo diversos efeitos biológicos como antitumoral, imunoprotetor, antinflamatório, neuroprotetor, antiviral e antilipemiante ( $\mathrm{Li}$ et al., 2016). Estes bioflavonóides podem atuar no nosso organismo como promotores de saúde devido ao seu amplo espectro biológico distinto, contribuindo para o aumento da capacidade física e mental (D'Andrea et al., 2015). A partir deste estudo sabe-se que estes compostos benéficos podem ser encontrados também nos frutos das PANC em concentrações de interesse.

$\mathrm{O}$ ácido ferúlico (AF) é um composto antioxidante encontrado geralmente nas folhas e sementes das plantas, especialmente farelo de milho e arroz, em todas as partes do trigo e aveia, café, maçã, alcachofra, amendoim, laranja e abacaxi (Chaudhary et al., 2019). Possui baixa toxicidade e diversas propriedades biológicas, como antimicrobiano, antidiabético, antinflamatórios e anticarcinogênico (Zduńska et al., 2018). Estudos mostram que o AF possui também um alto potencial biológico fotoprotetor, agindo como uma barreira na membrana celular, através da aplicação tópica, a qual, quando associada as vitaminas $\mathrm{C}$ e E, pode inibir a formação de eritema e carcinomas provocados pela exposição ao sol, diminuindo a absorção dos raios UVA e UVB. (Saija et al., 2000). Este composto também pode reduzir danos causados às células do fígado, reduzindo a fibrose hepática via inibição da sinalização do fator de transformação de crescimento $\beta 1$ (TGF- $\beta 1$ ) (Mu et al., 2018).

A naringina é um metabólito secundário que pode auxiliar o organismo contra afecções causadas pela SM (Síndrome Metabólica), diabetes tipo 2, obesidade, hipertensão, doenças cardiovasculares e, principalmente, doenças neurodegenerativas (Ahmed et al., 2019). Este composto inibe potencialmente a proliferação e secreção de lipoproteínas que contém apolipoproteína em hepatócitos e inibe a secreção das lipoproteínas VLDL, podendo auxiliar nos processos de dislipemias e reduzir o nível sérico na corrente sanguínea, contribuindo para diminuir a arterosclerose e a pressão arterial (Cho et al., 2011). Estudos recentes mostram também que a naringina pode contribuir para a redução da esteatose e lesão hepática alcóolica, reduzindo o estresse oxidativo e apoptose celular, controlando a liberação de citocinas pró-inflamatórias (Zhou et al., 2019).

O ácido gálico (AG) é um flavonóide derivado dos polifenóis encontrado em chás, uvas, vinho tinto, castanha entre outros produtos naturais, podendo ser obtido a partir da hidrólise ácida de taninos. Estudos com inflamação induzida por LPS (lipopolissacarídeos) em macrófagos RAW264.7, tratados com extrato de romã sugerem que a ação antinflamatória está 
diretamente relacionada a presença do ácido elágico e gálico, onde observou-se a inibição potencialmente da produção de óxido nítrico, PGE-2 (prostaglandina E2) e interleucinas IL-6 (BenSaad et al., 2017). Estudos epidemiológicos com o vírus influenza A (H1N1), indicam que o uso de ácido gálico oriundo da extração das folhas de Toonasinensis, sob concentração de 2,6 $\mu \mathrm{g} / \mathrm{mL}$, foi capaz de inibir a proliferação da infecção causada pela virulência por meio da regulação das moléculas de adesão e quimiocina que auxiliam na fixação viral (You et al., 2018).

A vitexina e seu isômero isovitexina são antioxidantes derivados das plantas que apresentam a capacidade de reduzir os efeitos deletérios do acidente vascular cerebral isquêmico, agindo sobre os níveis de estresse oxidativo, malondialdeído, óxido nítrico e citocinas pró-inflamatórias. (Jiang et al., 2018). Plantas dos gêneros Crataegus, Vitex, Passiflora e Echinodorus, são comumente utilizadas para o tratamento de alergias respiratórias e asma, seu uso pode estar associado a presença deste composto, pois estudos sugerem que a vitexina pode suprimir a hipersensibilidade alérgica em ratos induzida por ovalbumina, a qual pode ser utilizada para a produção de novos fármacos para tratamento de afecções respiratórias (Venturini et al., 2018).

Estudos têm mostrado que a rutina, que também faz parte dos flavonoides apresenta potencial antioxidante, o qual é capaz de diminuir a formação de sorbitol, espécies reativas de oxigênio, precursores da produtos finais de glicação avançada e citocinas inflamatórias (Ghorbani et al., 2017). Demais pesquisas sugerem que este composto pode auxiliar nas diversas patologias associadas ao diabetes Mellitus, como a nefropatia, dislipidemia, neuropatia e distúrbios cardiovasculares, estando associada a capacidade antioxidante, antinflamatório e organoprotetores (Ola et al., 2015). Por ser uma molécula glicosídica, a rutina têm capacidade de atravessar a membrana hematoencefálica, podendo exercer potencial terapêutico frente às doenças neurodegenerativas como depressão e Alzheimer, estando associada a agregação e ação da beta amilóide (A $\beta)$ e alteração do equilíbrio oxidante-antioxidante (Habtemariam, et al., 2016). Este composto também pode ser utilizado como cardioprotetor na sepse, pois é capaz de ativar o sistema enzimático endógeno da superóxido dismutase (SOD) e catalase (CAT), equilibrar a atividade do fator de necrose tumoral $\alpha(\mathrm{TNF}-\alpha)$ e interleucina 6 (IL-6), diminuindo a resposta inflamatória relacionada ao sistema cardiovascular (Xianchu et al., 2018). Sendo assim, agora sabe-se que estes compostos também podem ser encontradas nos frutos das espécies nativas negligenciadas consideradas PANC.

Com os resultados obtidos neste estudo, espera-se proporcionar o desenvolvimento social, econômico e ambiental da região, por meio da valorização das pequenas frutas e demais espécies nativas, gerando renda para os agricultores familiares. Buscamos também, conscientizar a população em geral frente a proteção ambiental e segurança alimentar por meio do consumo desta diversidade de espécies negligenciadas. Visamos ainda, a possibilidade na redução do uso excessivo de agroquímicos na Serra Gaúcha, para controle destas espécies consideradas inços, mas que através deste estudo prévio, sabemos agora que podem viabilizar a produção de nutracêuticos ou fármacos industriais, auxiliando na saúde da população em geral.

\section{Conclusão}

Este estudo mostrou que as três plantas ruderais estudadas, conhecidas como PANC, são boas fontes de compostos fenólicos, podendo ser utilizadas como antioxidantes. Frente aos resultados encontrados, consideramos a L. regnelli como fruto promissor para maiores análises futuras, tanto para atividade biológica, quanto para o desenvolvimento de novas formas de cultivo desta espécie. O potencial dos compostos fenólicos frente a regulação das disfunções mitocondriais relacionadas ao estresse oxidativo já são reportados em literatura logo, sugerimos que demais estudos acerca da atividade antioxidante in vitro e in vivo poderão ser realizados utilizando os extratos aquosos de L. regnelli, bem como, pesquisas relacionadas ao metabolismo redox celular, elucidando melhor os mecanismos moleculares envolvidos na função mitocondrial e estresse oxidativo. 


\section{Agradecimentos}

Agradecemos a Universidade de Caxias do Sul pela concessão do espaço para o desenvolvimento desta pesquisa e as agências de fomento CAPES e CNPq pelo apoio financeiro.

\section{Referências}

Ahmed, S., Khan, H., Aschner, M., Hasan, M.M. \& Hassan, S. T. S. (2019) Therapeutic potential of naringin in neurological disorders. Food Chemical Toxicoly. $132: 110646$.

Araújo, J. S., Chambó, E. D., Costa, M., Cavalcante da Silva, S., Lopes de Carvalho, C. A., \& Estevinho, L. (2017). Chemical Composition and Biological Activities of Mono- and Heterofloral Bee Pollen of Different Geographical Origins. International Journal Of Molecular Sciences, $18(5), 921$.

Baldermann, S., Blagojević, L., Frede, K., Klopsch, R., Neugart, S., Neumann, A., Ngwene, B., Norkeweit, J., Schröter, D., Schröter, A., Schweigert, F.J., Wiesner, M. \& Schreiner, M. (2016) Are Neglected Plants the Food for the Future? Critical Reviews in Plant Sciences, 35:2, 106-119.

Barbosa, T. P., Lins, J. A. S., Silva, G. M. da, Valente, E. C. N., \& Lima, A. S. T. (2021). Non-conventional food plants: are there potential consumers and places to buy them? Research, Society and Development, 10(4), e27710414146.

BenSaad L. A., Kim K. H., Quah C. C., Kim W. R. \& Shahimi M. (2017) Anti-inflammatory potential of ellagic acid, gallic acid and punicalagin A\&B isolated from Punica granatum. BMC Complementary Alternative Medicin, 14;17(1):47.

Boari, F., Cefola, M., Di Gioia, F., Pace, B., Serio, F. \& Cantore, V. (2013). Effect of cooking methods on antioxidant activity and nitrate content of selected wild Mediterranean plants. International Journal Of Food Sciences And Nutrition, 64(7), 870-876.

Corrêa, J., Bianchin, M., Lopes, A. P., Silva, E., Ames, F. Q., Pomini, A. M., Carpes, S. T., de Carvalho Rinaldi, J., Cabral Melo, R., Kioshima, E. S., BersaniAmado, C. A., Pilau, E. J., de Carvalho, J. E., Ruiz, A., Visentainer, J. V., \& Santin, S. (2021). Chemical profile, antioxidant and anti-inflammatory properties of Miconia albicans (Sw.) Triana (Melastomataceae) fruits extract. Journal Of Ethnopharmacology, $273,113979$.

Cunha, M. A. da, Pinto, L. C., Santos, I. R. P. dos, Neves, B. M., \& Cardoso, R. de C. V. (2021). Neglected and Underutilized Species in the perspective of Food and Nutritional Security promotion in Brazil. Research, Society and Development, 10(3), e20610313306.

Chaudhary A., Jaswal V. S., Choudhary S., Sonika S. A., Beniwal V., Tuli, H. S. \& Sharma S. (2019) Ferulic Acid: A Promising Therapeutic Phytochemical and Recent Patents Advances. Recent Patents on Inflammation \& Allergy Drug Discovery, 13(2):115-123.

Cheptou, P.-O., Carrue, O., Rouifed, S., \& Cantarel, A. (2008). Rapid evolution of seed dispersal in an urban environment in the weed Crepis sancta. Proceedings of the National Academy of Sciences, 105: 3796-3799.

Cho, K.W., Kim, Y.O., Andrade, J.E., Burgess, J.R. \& Kim, Y.-C. (2011). Dietary naringenin increases hepatic peroxisome proliferators-activated receptor $\alpha$ protein expression and decreases plasma triglyceride and adiposity in rats. European Journal of Nutrition, 50, 81-88.

Dai J. \& Mumper R. J. (2010) Plant phenolics: extraction, analysis and their antioxidant and anticancer properties. Molecules, 15(10):7313-7352.

D'Andrea G. (2015) Quercetin: A flavonol with multifaceted therapeutic applications. Fitoterapia. 106, $256-271$.

D'Archivio M., Filesi C., Di Benedetto R., Gargiulo R., Giovannini C. \& Masella R. (2007) Polifenóis, fontes dietéticas e biodisponibilidade. Annali dell'Istituto Superiore di Sanità, 43:348-361.

Del Tredici, P. (2010). Wild urban plants of the Northeast. Cornell University Press, Ithaca. Book.

Di Lorenzo, C., Colombo, F., Biella, S., Stockley, C., \& Restani, P. (2021). Polyphenols and Human Health: The Role of Bioavailability. Nutrients, 13(1), 273.

Du L, Wang J. X, Chen C. D., Wang F., Zhou H. Y. \& Du W. (2018) Triterpenoid saponins from roots of Phytolacca acinosa. Zhongguo Zhong Yao Za Zhi. 43(12):2552-2556.

Ferreira-Gomes, L., Nascimento-Martins, D. H., Mazutti, S. M., de Barros, Y. Y., de Souza, P. M., Freitas, M. M., Fagg, C. W., Simeoni, L. A., Magalhães, P. O., Silveira, D., \& Fonseca-Bazzo, Y. M. (2021). Propriedades biológicas y caracterización fitoquímica del extracto acuoso de Miconia chamissois Naudin. Boletín Latinoamericano y del Caribe de Plantas Medicinales y Aromáticas, 20(4), 427-442.

Fereidoon Shahidi, P. K. Janitha \& P. D. Wanasundara (1992) Phenolic antioxidants, Critical Reviews in Food Science and Nutrition, 32:1, 67-103.

Filippin, L. I., Vercelino R., Marroni N. P. \& Xavier R. M. (2008) Influência de processos redox na resposta inflamatória da artrite reumatóide. Revista Brasileira de Reumatologia, 48(1)17-24.

Ghorbani A. (2017) Mechanisms of antidiabetic effects of flavonoid rutin. Biomedicine \& Pharmacotherapy, 96:305-312.

Habtemariam S. \& Lentini G. (2015) The therapeutic potential of rutin for diabetes: an update. Mini-Reviews in Medicinal Chemistry, 15(7):524-8.

Habtemariam S. (2016) Rutin as a Natural Therapy for Alzheimer's Disease: Insights into its Mechanisms of Action. Current Medicinal Chemistry, 23(9):86073.

Hameed, A., \& Akhtar, N. (2018). Comparative chemical investigation and evaluation of antioxidant and tyrosinase inhibitory effects of Withania somnifera (L.) Dunal and Solanum nigrum (L.) berries. Acta Pharmaceutica (Zagreb, Croatia), 68(1), 47-60. 
Jiang J, Dai J, \& Cui H. (2018) Vitexin reverses the autophagy dysfunction to attenuate MCAO-induced cerebral ischemic stroke via mTOR/Ulk1 pathway. Biomedicine \& Pharmacotherapy, 99:583-590.

Khan, H. J., Ahmad, M. K., Khan, A. R., Rastogi, N., Mahdi, A. A., Ansari, J. A., Fatima, N., \& Satyanarayan, G. (2016). Identification of Anticancer and Antioxidant phytoconstituents from chloroform fraction of Solanum nigrum L. berries using GC-MS/MS analysis. Indian Journal Of Experimental Biology, 54(11), 774-782.

Kaunda, J. S., \& Zhang, Y. J. (2019). The Genus Solanum: An Ethnopharmacological, Phytochemical and Biological Properties Review. Natural Products And Bioprospecting, 9(2), 77-137.

Kinupp V. F. \& Barros I.B.I.D. (2007) Riqueza de plantas alimentícias não-convencionais na região metropolitana de Porto Alegre, Rio Grande do Sul. Revista Brasileira de Biociências 5: 63-65.

Leal M. L, Alves R.P. \& Hanazaki N. (2018) Knowledge, use, and disuse of unconventional food plants. Journal of Ethnobiology and Ethnomedicine, $14: 6$.

Li Y, Yao J, Han C. (2016) Quercetin, Inflammation and Immunity. Nutrients, 8(3):167.

Ma X. P., Zhang W.F., Yi P., Lan J.J., Xia B., Jiang S., Lou H.Y. \& Pan W.D. (2017) Novel Flavones from the Root of Phytolacca acinosa Roxb. Chemistry \& Biodiversity, 14(12).

Maphosa Y. \& Jideani V.A. (2017) The role of legumes in human nutrition. In: Hueda MC (eds) Functional Food - Improve Healththrough Adequate Food, 10.5772 .

Meng, X., Li, Y., Lu, C., Zhao, M., Li, M., Wang, S., Zhao, C., Lin, B., Shang, L., Chu, Z., \& Ding, X. (2020). Purification and antioxidant capacity analysis of anthocyanin glucoside cinnamic ester isomers from Solanum nigrum fruits. Journal Of Separation Science, 43(12), 2311-2320.

Morais, M. G., Saldanha, A. A., Rodrigues, J. P. C., Mendes, I. C., Ferreira, L. M., Amado, P. A., Farias, K. S., Zanuncio, V. S. S., da Silva, D. B., Pinto, F. C. H., Soares, A. C., \& Lima, L. A. R. S. (2020). Chemical composition, antioxidant, anti-inflammatory and antinociceptive activities of the ethanol extract of ripe fruits of Solanum lycocarpum St. Hil. (Solanaceae). Journal Of Ethnopharmacology, 262, 113125.

Morelli, L.L.L., (2010), Avaliação de compostos fenólicos majoritários em geleia de uva produzida com a variedade IAC-138-22 (máximo). Dissertação de Mestrado. Universidade Estadual de Campinas. Campinas, SP, 133p.

Mu M., Zuo S., Wu R. M., Deng K. S., Lu S., Zhu J. J., Zou G. L., Yang J., Cheng M. L. \& Zhao X. K. (2018) Ferulic acid attenuates liver fibrosis and hepatic stellate cell activation via inhibition of TGF- $\beta /$ Smad signaling pathway. Drug Design, Development and Therapy, 3;12:4107-4115.

Nabavi S. M., Ebrahimzadeh M. A., Nabavi S. F., \& Bahramian F. (2009) In vitro antioxidant activity of Phytolacca americana berries. Pharmacology, 1:81-8. Nascimento V.T., Lucena R.F. Albuquerque U. P. \& Maciel M.I. (2013) Knowledge and use of wild food plants in areas of dry seasonal forests in Brazil. Ecology of Food and Nutrition, 52: 317-43.

Neergheen V.S., Soobrattee M.A., Bahorun T. \& Aruoma O.I. (2006) Caracterização dos constituintes fenólicos em plantas endêmicas da Ilha Maurícia como determinantes de suas atividades antioxidantes in vitro. Journal of Plant Physiology, 163:787-799.

Ola M.S., Ahmed M.M., Ahmad R., Abuohashish H.M., Al-Rejaie S.S. \& Alhomida A.S. (2015) Neuroprotective Effects of Rutin in Streptozotocin-Induced Diabetic Rat Retina. Journal of Molecular Neuroscience, 56(2):440-8.

Pereira, A. S., Shitsuka, D. M., Parreira, F. J., \& Shitsuka, R. (2018). Metodologia da pesquisa cientifica. [e-book]. Santa Maria: UAB / NTE / UFSM. https://www.ufsm.br/app/uploads/sites/358/2019/02/Metodologia-da-Pesquisa-Cientifica_final.pdf.

Petropoulos, S.A., Karkanis, A., Martins, N., \& Ferreira, I.C.F.R. (2018) Edible halophytes of the Mediterranean basin: Potential candidates for novel food products. Trends in Food Science and Technology, 74, 69-84.

Prakash, D., \& Sharma, G. (2014) Role of antioxidant polyphenols in nutraceuticals and human health. In: Prakash D, Sharma G (ed) Phytochemicals Of Nutraceutical Importance. CABI, Wallingford, UK, pp 208-228.

Quintans-Júnior, L. J., Gandhi, S. R., Passos, F., Heimfarth, L., Pereira, E., Monteiro, B. S., Dos Santos, K. S., Duarte, M. C., Abreu, L. S., Nascimento, Y. M., Tavares, J. F., Silva, M. S., Menezes, I., Coutinho, H., Lima, A., Zengin, G., \& Quintans, J. (2020). Dereplication and quantification of the ethanol extract of Miconia albicans (Melastomaceae) by HPLC-DAD-ESI-/MS/MS, and assessment of its anti-hyperalgesic and anti-inflammatory profiles in a mice arthritis-like model: Evidence for involvement of TNF- $\alpha$, IL-1 $\beta$ and IL-6. Journal Of Ethnopharmacology, 258, 112938.

Ribani M., Bottoli C.B.G., Collins C.H., Jardim I.C.S.F. \& Melo L.F.C. (2004) Validação em métodos cromatográficos e eletroforéticos. Química Nova, 27, $771-780$.

Saija A, Tomaino A, Trombetta D, De Pasquale A, Uccella N, Barbuzzi T, Paolino D. \& Bonina F. (2000) In vitro and in vivo evaluation of caffeic and ferulic acids as topical photoprotective agents. International Journal of Pharmaceutics, 10;199(1):39-47.

Santos-Buelga, C., González-Paramás, A. M., Oludemi, T., Ayuda-Durán, B., \& González-Manzano, S. (2019). Plant phenolics as functional food ingredients. Advances In Food And Nutrition Research, 90, 183-257.

Tufarelli, V., Casalino, E., D'Alessandro, A. G., \& Laudadio, V. (2017). Dietary Phenolic Compounds: Biochemistry, Metabolism and Significance in Animal and Human Health. Current Drug Metabolism, 18(10), 905-913.

Ullah, N., Haq, I. U., Safdar, N., \& Mirza, B. (2015). Physiological and biochemical mechanisms of allelopathy mediated by the allelochemical extracts of Phytolacca latbenia (Moq.) H. Walter. Toxicology And Industrial Health, 31(10), 931-937. 
Research, Society and Development, v. 10, n. 17, e190101724210, 2021

(CC BY 4.0) | ISSN 2525-3409 | DOI: http://dx.doi.org/10.33448/rsd-v10i17.24210

Venturini C.L., Macho A., Arunachalam K., de Almeida D.A.T, Rosa S.I.G., Pavan E., Balogun S.O., Damazo A.S. \& Martins D.T.O. (2018) Vitexin inhibits inflammation in murine ovalbumin-induced allergic asthma. Biomedicine \& Pharmacotherapy, 97:143-151.

Xianchu L, Lan Z, Ming L, Yanzhi M. (2018) Protective effects of rutin on lipopolysaccharide-induced heart injury in mice. The Journal of Toxicological Sciences, 43(5):329-337.

Xiang, L., Wang, Y., Yi, X., \& He, X. (2019). Steroidal alkaloid glycosides and phenolics from the immature fruits of Solanum nigrum. Fitoterapia, $137,104268$.

Xu B.J., Chang S.K. (2007) A comparative study on phenolic profiles and antioxidant activities of legumes as affected by extraction solvents. Journal of Food Science, 72:S159-166.

Yang, L., Gao, S., Su, Z., Qin, X., \& Li, Z. (2021). Identification of the constituents and the cancer-related targets of the fruit of Solanum nigrum based on molecular docking and network pharmacology. Journal Of Pharmaceutical And Biomedical Analysis, $200,114067$.

You, H. L., Huang, C. C., Chen, C. J., Chang, C. C., Liao, P. L. \& Huang, S. T. (2018) Anti-pandemic influenza A (H1N1) virus potential of catechin and gallic acid. Journal of the Chinese Medical Association, 81(5):458-468.

Zduńska, K., Dana, A., Kolodziejczak, A., \& Rotsztejn, H. (2018) Antioxidant Properties of Ferulic Acid and Its Possible Application. Skin Pharmacology and Physiology, 31(6):332-336.

Zheleva-Dimitrova D. (2013). Antioxidant and acetylcholinesterase inhibition properties of Amorpha fruticosa L. and Phytolacca americana L. Pharmacognosy Magazine, 9(34), 109-113.

Zhou, C., Lai, Y., Huang, P., Xie, L., Lin, H., Zhou, Z., Mo, C., Deng, G., Yan, W., Gao, Z., Huang, S., Chen, Y., Sun, X., Lv, Z., \& Gao L. (2019) Naringin attenuates alcoholic liver injury by reducing lipid accumulation and oxidative stress. Life Sciences, 1;216:305-312. 